Check for updates

Cite this: J. Mater. Chem. C, 2019, 7, 13502

\section{Unravelling the photothermal and photomechanical contributions to actuation of azobenzene-doped liquid crystal polymers in air and water $\dagger$}

\author{
Marina Pilz da Cunha, (D) Evelien A. J. van Thoor, (D) Michael G. Debije, (DD \\ Dirk J. Broer (D) and Albert P. H. J. Schenning (D)*
}

\begin{abstract}
Liquid crystal networks doped with azobenzene molecular photo-switches have been commonly used as responsive polymers in untethered soft/micro robots. Understanding the underlying actuation mechanism of azobenzene chromophores in aqueous and ambient environments is crucial for the control of deformation speed and amplitude of the photo responsive actuators. Here, a systematic study is presented that clarifies the outstanding uncertainty in the mechanistic contributions of azobenzene powered actuation. The photothermal and photomechanical contributions in the mechanism are elucidated and the mechanistic dependence on azobenzene chemistry is demonstrated. By connecting mechanical, thermal, isomerization and actuation analysis in both dry and aqueous environments, this study reveals design guidelines for light-responsive amphibious soft robots.
\end{abstract}

Received 11th August 2019, Accepted 14th October 2019

DOI: $10.1039 /$ c9tc04440j

rsc.li/materials-c

\section{Introduction}

Over the last decade there has been an increasing interest in stimuli-responsive materials for a multitude of applications including smart textiles, ${ }^{1}$ drug delivery ${ }^{2}$ microfluidics ${ }^{3}$ and soft robotics. ${ }^{4-6}$ of particular interest are soft materials presenting mechanical deformation as a response to remotely addressed stimuli, including light, ${ }^{7,8}$ magnetic fields, ${ }^{9}$ humidity, ${ }^{10,11}$ and pH. ${ }^{12}$ As an untethered stimulus, light is commonly employed due to ease of application, high degree of tunability and temporal resolution. Liquid crystal networks (LCNs) are a prevalent class of soft smart materials, capable of functioning in dry environments with directed molecular self-assembly allowing for tuneable and complex nano-scale molecular designs. A prominent choice of photo-switch in light responsive LCNs are azobenzene derivatives due to their facile incorporation into the liquid crystalline network without disruption of molecular alignments. ${ }^{13}$ Photo responsivity is achieved through the reversible isomerization of the rod-like, stable trans azobenzene isomer into the bent cis isomer, accompanied by a shift in absorbance. LCNs containing azobenzene photo-switches have been designed to perform remotely controlled bending, ${ }^{14}$ curling $^{15}$ and more complex oscillatory motion activated by light. ${ }^{16-18}$ Transforming light into mechanical motion has led to the development of

Laboratory of Stimuli-Responsive Functional Materials \& Devices, Department of Chemical Engineering and Chemistry, Eindhoven University of Technology, P. O. Box 513, 5600 MB, Eindhoven, The Netherlands. E-mail: a.p.h.j.schenning@tue.nl

$\dagger$ Electronic supplementary information (ESI) available. See DOI: 10.1039/c9tc04440j
LCN-based soft robots displaying inchworm-like walking, ${ }^{19,20}$ rolling $^{21-23}$ and caterpillar-like ${ }^{24,25}$ locomotion in dry environments and a recent endeavour is to expand the locomotion into water-based environments through the development of microswimmers. $^{26,27}$

Elucidation of the complex mechanisms for photoinduced mechanical deformations observed in azobenzene-based LCN cantilevers has been extensively pursued in the literature, but an expanding library of azobenzene derivatives has left large uncertainties in uncovering mechanistic guidelines as to which azobenzene moiety to select for specific applications. Current literature suggests that the light-triggered actuation of LCNs containing azobenzene chromophores is rooted in both photomechanical and photothermal effects. Photomechanical ${ }^{28}$ factors are derived from isomerization-induced effects which include molecular order disruption ${ }^{29,30}$ and free-volume formation ${ }^{31,32}$ as a result of the cis isomer bent conformation. Additionally, the diminished molecular length of the azobenzene moiety from 9 to $5.5 \AA,^{33}$ corresponding to trans and cis isomers respectively, can generate an effective isomer-generated network stress (pulleffects). ${ }^{34}$ Photothermal effects include isomerization-driven heating and network photo-softening, ${ }^{35-37}$ which are responsible for the large light-induced storage modulus decrease that significantly surpasses the commonly observed thermal-softening of glassy polymers. ${ }^{38}$ Understanding the interplay of molecular processes, mechanical and actuating properties will be crucial in advancing the field of soft robotics. An ambiguity remains in understanding of how the mechanism for photoinduced 
deformation differs between photo-switches of different chemical natures. Additionally, the molecular requirements for a photoswitch to induce actuation in both dry and aqueous environments is still largely concealed. Creation of such guidelines will be of great importance for the development of amphibious-like actuators. To date, no study has connected isomerization kinetic data, mechano-thermal tests with actuation amplitudes and rates to investigate which photomechanical or photothermal effects become dominant in the deformation mechanism of photoswitches of differing chemical natures in either air or aqueous environments.

We present a systematic study highlighting the nature of the azobenzene photo-switch and how it impacts the actuation mechanism. The networks investigated are splay aligned, containing low ( $2 \mathrm{~mol} \%$ ) concentrations of different photo-active azobenzene derivatives. Actuation is achieved through illumination using a collimated light source, leading the polymer films to undergo an uncurling motion directed towards the light source, Fig. 1A. Our choice of azobenzene moieties include two of the most popularly used derivatives for actuator fabrication, photoswitch DY and MR, Fig. 1C. We explore the effect of fully and partially attaching the chromophore to the network by comparing diacrylate and monoacrylate azobenzene moieties, as well as study the effect of cis isomer lifetime on actuation. Furthermore, this study is expanded to include actuation in aqueous environments. For the first time, we show a combined study that connects mechanical, thermal and actuation analysis with isomerization kinetic studies to uncover the relative contributions of photothermal and photomechanical effects to macroscopic bending in splay aligned liquid crystal cantilevers. This study provides essential guidelines for future designs of light responsive actuators by aiding the choice of azobenzene derivative most appropriate for each application.

\section{Results and discussion}

\section{Preparation of the liquid crystal networks}

The photo-responsive acrylate-based LCNs are composed of two liquid crystal mesogens (1 and 2, Fig. 1B) and $2 \mathrm{~mol} \%$ of an azobenzene derivative. The azobenzene naming is based on the crosslink nature of the molecule: mono- (M) or di- (D) acrylate and on the color of the film, either yellow (Y) or red (R). The azobenzenes used were MY (monoacrylate azobenzene with a long cis lifetime (hours) see Fig. S1 (ESI $\dagger$ ): the films have a yellow color), DY (diacrylate azobenzene with long cis lifetime (hours): films have has a yellow color) or MR (monoacrylate azobenzene with short cis lifetime: films have a red color), ${ }^{39}$ Fig. 1C. Crosslink density is equal in all mixtures, adjusted by altering the content of crosslinker 1 in the film containing

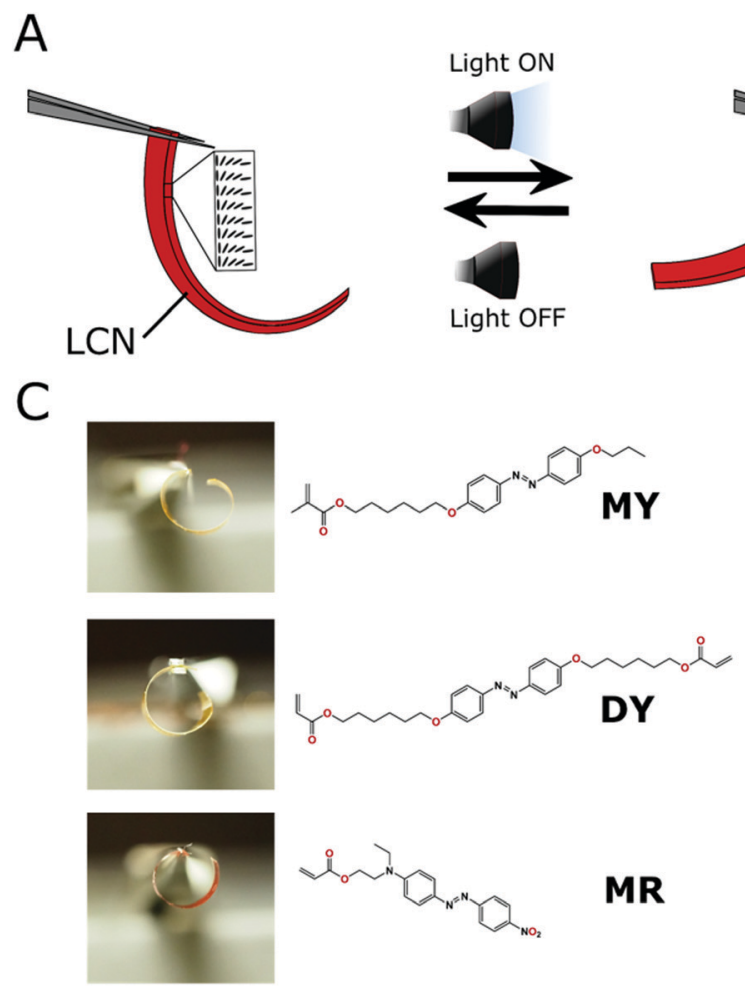

B

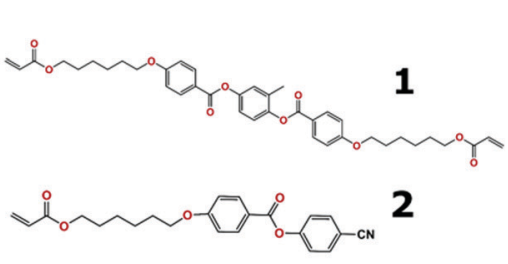

D

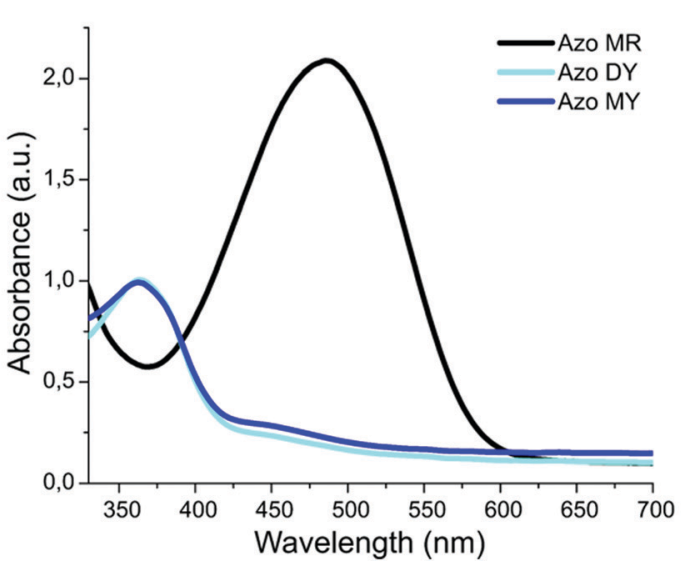

Fig. 1 (A) The splay liquid crystal networks have a pre-bent shape at room temperature, with the homeotropic surface on the inside of the curl. Upon illumination the film shows unbending into a flattened geometry: the actuation is reversible. (B) The molecular structure of the liquid crystalline mesogens composing the LCN. (C) Photograph of the three different azobenzene-doped films investigated; MY and DY both have a yellow color and MR has an overall red appearance. Before illumination and at room temperature, all films have a pre-bent shape. (D) Absorption spectra of the three splay networks before light exposure; all films contain 2 mol\% of the azobenzene derivative. 
diacrylate azobenzene (mixture compositions in Table S1, ESI $\dagger$ ). The network is photo-polymerized in a $20 \mu \mathrm{m}$ gap glass cell filled with the liquid crystal (LC) mixture by capillary action. The cell employs homeotropic and planar alignment layers on either face of the cell for the realization of an overall splay alignment within the film. Polymerization is carried out at $80{ }^{\circ} \mathrm{C}$ in the nematic LC phase (see Fig. S2, ESI $\dagger$ ). A subsequent thermal post-cure at $120{ }^{\circ} \mathrm{C}$ ensures full polymerization and releases mechanical stresses generated from polymerization shrinkage. For photo-polymerization of films containing azobenzene derivatives MY and DY, a filter was used to block wavelengths below $400 \mathrm{~nm}$, preventing excessive trans-cis isomerization during polymerization.

After opening the glass cells, the polymer films are cut into $5 \times 20 \mathrm{~mm}^{2}$ strips, with the molecular director of the LC molecules parallel to the strip length. At room temperature, the splay films have a pre-curled shape, with the homeotropic side of the splay on the inside of the curl. The pre-curl is due to an increased molecular order at lower temperatures: cooling from the elevated polymerization temperature $\left(80{ }^{\circ} \mathrm{C}\right)$ causes anisotropic molecular volume contraction and expansion, parallel and perpendicular to the molecular director, respectively (Fig. S3, $\mathrm{ESI} \dagger$ ). The splay aligned networks all show storage moduli (direction parallel to the planar aligned molecules) in the range of 1.2-1.8 GPa and glass transition temperatures $\left(T_{\mathrm{g}}\right)$ around $80{ }^{\circ} \mathrm{C}$.

LCNs doped with azo-MR show a single intense absorption peak centered around $450 \mathrm{~nm}$, Fig. 1D. The absence of a secondary peak corresponding to a cis absorption band is due to the derivative's very short cis lifetime, a result of the effective electron push-pull configuration generated by the ring substituted electron donating and accepting groups on opposing sides of the aromatic system. The other two azobenzene moieties, MY and DY, show equivalent extinction coefficients, seen by equal absorbance profiles (Fig. 1), and have cis isomers with longer lifetimes, 4 and $1.5 \mathrm{~h}$ in the dark, respectively, Fig. S1 (ESI $\dagger$ ). The shorter cis lifetime of DY in the dark is likely due to the crosslinker nature of the moiety, facilitating the return to the stable trans state by the overall network. The trans state absorbance of both DY and MY is dominated by the $\pi-\pi^{*}$ absorption peak centered around $365 \mathrm{~nm}$ and a lower $\mathrm{n}-\pi^{*}$ absorption at around $450 \mathrm{~nm}$. Isomer trans-cis conversion is visualized by a decrease in the absorption at $365 \mathrm{~nm}$ and an increase at $450 \mathrm{~nm} .^{40}$

\section{Photo-responsive actuation}

Actuation in air. Splay aligned LCNs are commonly used as stimuli-responsive cantilevers due to large macroscopic actuation in which the amplitude is directly controlled by the stimulus intensity and motion reversibility can be achieved upon removal of the stimulus. ${ }^{41,42}$ Actuation can be triggered through illumination with wavelengths absorbed by either the trans or cis state of the photo-switch. In this study, actuation of MR-azo doped films is studied through illumination with $455 \mathrm{~nm}$ light. For MY- and DY-azo films, illumination with either 455 or $365 \mathrm{~nm}$ light is investigated due to the relatively stable cis state, which results in two distinct absorption bands centered around 365 (trans state) and 450 (cis state). Upon illumination, the three films show fast unbending actuation directed toward the light source, demonstrated by the overlay of figures during the motion of forward actuation, Fig. 2A. All films remain in the unbent state until removal of the light stimulus. Once the light is turned off, films containing side-chain chromophores, MR and MY, immediately return to the initial pre-curled position, Fig. 2B. The timeline for actuation of the films upon illumination with $455 \mathrm{~nm}$ (at $\left.310 \mathrm{~mW} \mathrm{~cm}^{-2}\right)$ and $365 \mathrm{~nm}\left(170 \mathrm{~mW} \mathrm{~cm}^{-2}\right)$ are presented in Fig. 2C. The reverse actuation for crosslinked azobenzene-DY follows a very different timescale after illumination with $365 \mathrm{~nm}$ light. Immediately upon removal of the $365 \mathrm{~nm}$ light, the film shows only minor re-curling (Fig. $2 \mathrm{~B}$ and $\mathrm{C}$ ), with full return to the pre-bent state observed only after hours. The timescale for the macroscopic recovery to the pre-bent state does not match

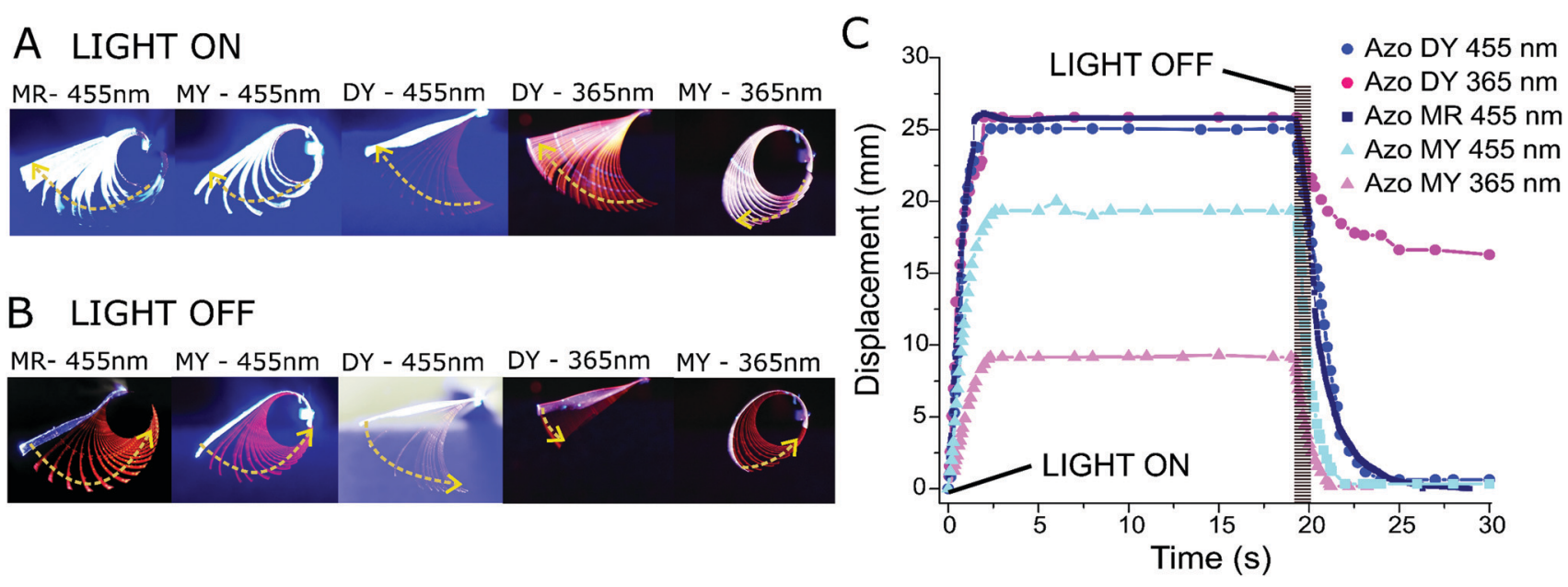

Fig. 2 (A) Stacked images of the forward actuation of splay LCNs containing azobenzene MR, DY or MY, respectively, in air upon exposure to either 455 ( $310 \mathrm{~mW} \mathrm{~cm}^{-2}$ ) or $365\left(170 \mathrm{~mW} \mathrm{~cm}{ }^{-2}\right) \mathrm{nm}$; light is incident from the left. The samples are illuminated after a long period of storage in the dark. Arrows indicate the direction of actuation. (B) Stacked images of the reverse actuation during the first 30 seconds after removal of light. (C) Timescale of the tipdisplacement of the films upon illumination and upon removal of the light stimulus, either $365 \mathrm{~nm}\left(170 \mathrm{~mW} \mathrm{~cm}^{-2}\right)$ or $455 \mathrm{~nm}\left(310 \mathrm{~mW} \mathrm{~cm}{ }^{-2}\right)$ light. 
the trans isomer population recovery, Fig. S4 (ESI $\dagger$ ). The mismatch between macroscopic shape recovery and isomerization kinetics has been previously reported for dynamic surfaces. ${ }^{31}$ On the other hand, after illumination with $455 \mathrm{~nm}$, the films containing crosslinked azobenzene show complete re-curling on a timescale similar to the side-chain photo-switches. From Fig. 2C we observe a curious difference in the actuation potential between the two yellow films (MY and DY) when illuminated with $365 \mathrm{~nm}$ light. To investigate the actuation dependence of the films on the light intensity and wavelength of illumination, we present a systematic analysis that combines thermal and displacement tracking during actuation, Fig. 3.

The extent of actuation of the LCNs is observed to be heavily dependent on the light intensity used for actuation. Additionally, at equal light intensities, films containing different azobenzene moieties show strikingly different deformation amplitudes. The actuation efficiency for the individual films is illustrated as the maximum film tip displacement at varied illumination intensities of 365 or $455 \mathrm{~nm}$ light, Fig. 3A. Splay films doped with chromophore DY clearly display superior actuation efficiency upon illumination with $365 \mathrm{~nm}$ light: exposure of the same film to $455 \mathrm{~nm}$ light results in a slightly lowered actuation efficiency. On the other hand, actuation of MY doped films shows nearly no wavelength dependence, with both 365 and $455 \mathrm{~nm}$ illumination resulting in similar displacements. All films show a temperature increase when illuminated, the increase being linearly correlated to the incident light energy, Fig. 3B. The temperature increases caused by illumination of DY and MY films are identical, with a maximum temperature of $65{ }^{\circ} \mathrm{C}$ at $455 \mathrm{~nm}, 310 \mathrm{~mW} \mathrm{~cm}^{-2}$. This correlation is likely due to the equivalent absorption spectra of the two chromophores (Fig. 1C). The temperature increase observed is connected to the percentage light energy absorbed by the chromophore. In this way, one could expect $365 \mathrm{~nm}$ to lead to a higher film temperature as the trans state shows large spectrum absorption of the $365 \mathrm{~nm}$ LED (reaching above 80\% absorption, see Experimental section for details). However, upon $365 \mathrm{~nm}$ illumination, fast trans-cis conversion (Fig. S1, ESI $\dagger$ ), results in a dominant cis population, which absorbs only $60 \%$ of incident light. This absorption is similar to the $50 \%$ absorbed by the trans state at $455 \mathrm{~nm}$, leading to a near wavelength independence of energy absorbed. The higher film temperatures reached for MR azobenzene $\left(73{ }^{\circ} \mathrm{C}\right.$ at $\left.310 \mathrm{~mW} \mathrm{~cm}{ }^{-2}\right)$ is a consequence of the dye's greater light absorbance, i.e. higher extinction coefficient (Fig. 1C). Plotting the amplitude of displacement of the MR film against film temperature also shows a linear relationship, Fig. 3C. This plot demonstrates the near equivalent behavior of MY and MR doped films, suggesting that thermal effects play the governing role in the actuation mechanism of monoacrylate azobenzene chromophores.

Actuation in water. The most remarkable difference between the actuation potential of the three investigated azobenzene derivatives is observed in underwater actuation of the LCN containing crosslinker azo-DY. MY-azo films show very limited (Fig. S5, ESI $\dagger$ ) and MR-azo films show no actuation underwater at all. Starting from a non-illuminated, curled resting state, illumination of the submerged DY film (water at $18{ }^{\circ} \mathrm{C}$ ) with $365 \mathrm{~nm}$ light causes significant unbending towards the light source. Illumination with $455 \mathrm{~nm}$ results in very limited unbending actuation, Fig. 4A, contrasting with observations in dry environments (Fig. 2A, vide supra). Removal of the light does not result in the return to the film's initial curled shape (yellow striped curve in Fig. 4B): recovery of the pre-bent state after $365 \mathrm{~nm}$ illumination only takes place after a few hours in the dark. Alternatively, illumination with $455 \mathrm{~nm}$ light causes a rapid return to a bent state, Fig. 4C: however, the curl is not as pronounced as the initial pre-bend, which can only be recovered after relaxation in dark.

The speed of underwater actuation with $365 \mathrm{~nm}$ is considerably slower than for actuation in air with similar light intensities (Fig. 4C). Underwater, the cantilever takes around 30 seconds to reach maximum unbending deformation, whereas in air maximum uncurling is achieved in the first 2 seconds of illumination. Likewise, the amplitude of underwater deformation of DY-azo films is significantly smaller than in air and appears to only slightly increase at intensities beyond $40 \mathrm{~mW} \mathrm{~cm}^{-2}$, Fig. 4D. This behavior is remarkably different than actuation in-air, which appears to increase with incident light energies, reaching a maximum flattening geometry at

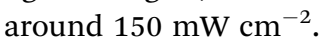
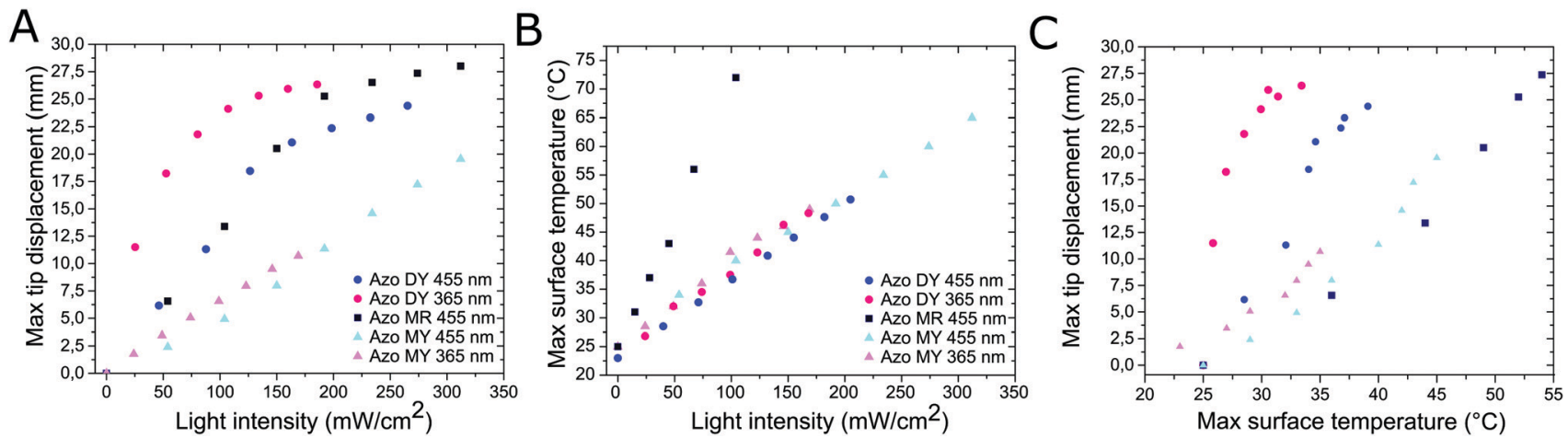

Fig. 3 (A) Maximum amplitude of actuation of the film's end-tip at varying light intensities for all azobenzene-doped films. (B) Maximum measured surface temperature of the films when illuminated with varying intensities of light. (C) Maximum amplitude of actuation of the film's end-tip as a function of the maximum measured surface temperature of the films. 
A LIGHT ON

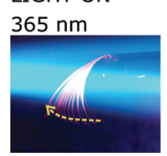

C

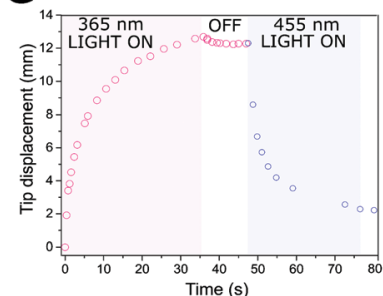

B LIGHT OFF after $365 \mathrm{~nm}$ illumination $365 \mathrm{~nm}$

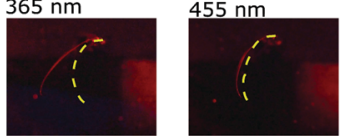

D

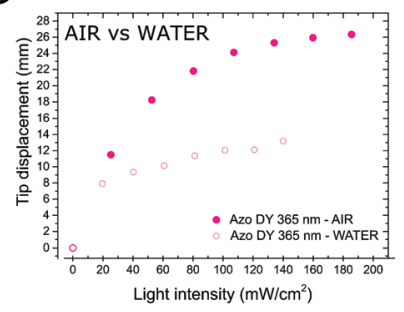

Fig. 4 (A) Stacked images of underwater actuation of a splay LCN containing DY azobenzene with 365 or $455 \mathrm{~nm}$ light after storage in dark; light incident from left. (B) Removal of light results in no reverse actuation, but a retention of the actuated state. (C) Timescale of underwater actuation of DY-azo film upon illumination with $140 \mathrm{~mW} \mathrm{~cm}^{-2} 365 \mathrm{~nm}$ light, switching of the light and $230 \mathrm{~mW} \mathrm{~cm}{ }^{-2} 455 \mathrm{~nm}$ light. (D) Maximum displacement for splay DY-azo LCN film in water and in dry environment upon illumination with $365 \mathrm{~nm}$ light at different intensities, illumination time is $30 \mathrm{~s}$ for air actuation and 3 minutes for in water actuation. The light intensity incident on the LCN under water is diminished when compared to actuation in air. This is due to water absorption and has been measured and considered.

\section{Mechanical, isomerization and thermal analysis}

Upon illumination, azo-doped LCNs experience alterations in photo-switch isomer population, film temperature and mechanical properties. ${ }^{36,37}$ To study the inter-relation of these factors during illumination of films in a dry environment, the films are first irradiated with $455 \mathrm{~nm}$ for 3 minutes to ensure near-equivalent trans state populations, which we denote as a relative population of $100 \%$ trans. Equivalently, we denote $0 \%$ trans as the resulting absorbance profile after $3 \mathrm{~min}$ high intensity $365 \mathrm{~nm}$ illumination. Fig. 5 displays the effect of illumination of the splay films doped with MY and DY azobenzene derivatives with both 365 and $455 \mathrm{~nm}$, considering sample temperature, storage modulus and isomer population. The same analysis is conducted for the MR derivative with single wavelength LED (455 nm), Fig. S6 (ESI $\dagger$ ). Fig. 5 illustrates that illumination with $365 \mathrm{~nm}$ light promotes trans-cis isomerization and a rapid decrease in the trans population is observed for both azo-moieties (Fig. 5A and B). The isomer population is observed to remain constant when the light is turned off, as both chromophores show relatively long-lived cis isomers, Fig. S1 (ESI $\dagger$ ). Accompanying the light triggered trans-cis isomerization, we observe an immediate increase in the film's surface temperature. Even though a constant trans population is established within the first few seconds of illumination, the film's temperature remains constant during illumination, as both cis and trans states show absorbance at 455 and $365 \mathrm{~nm}$ (Fig. 1C, vide supra). Ceasing illumination results in rapid cooling of the films. These observations suggest that film heating is independent of the population ratio between cis and trans isomers.

Connection between molecular processes and macroscopic properties is observed by tracking the storage modulus of the films during the illumination process. Upon illumination, the polymer films show a decrease in the storage modulus concurrent with the temperature increase; higher light intensities result in greater modulus decrease. The plasticization of the network by excitation of the photo-switches has been described as the photo-softening effect. The three azo-benzene derivatives investigated in this work show similar degrees of softening, with no obvious wavelength dependence (see Fig. S7, ESI $\dagger$ ). For both mono- and diacrylate chromophores, the storage modulus recovery upon turning off the excitation light correlates to the rapid temperature decrease of the film and not to the much slower isomer population reversion. Thus, we disconnect the network's mechanical properties from specific isomer populations and relate the material softening to the isomerization kinetics via temperature evolution. These observations suggest that photothermal effects such as network softening through isomerization of the azobenzene moieties are present in both mono- and diacrylate photo-switches.

\section{Mechanisms for light triggered actuation}

The mechanism for light-triggered actuation of LCNs containing azobenzene chromophores has roots in both photothermal and photomechanical factors. The contribution from each effect
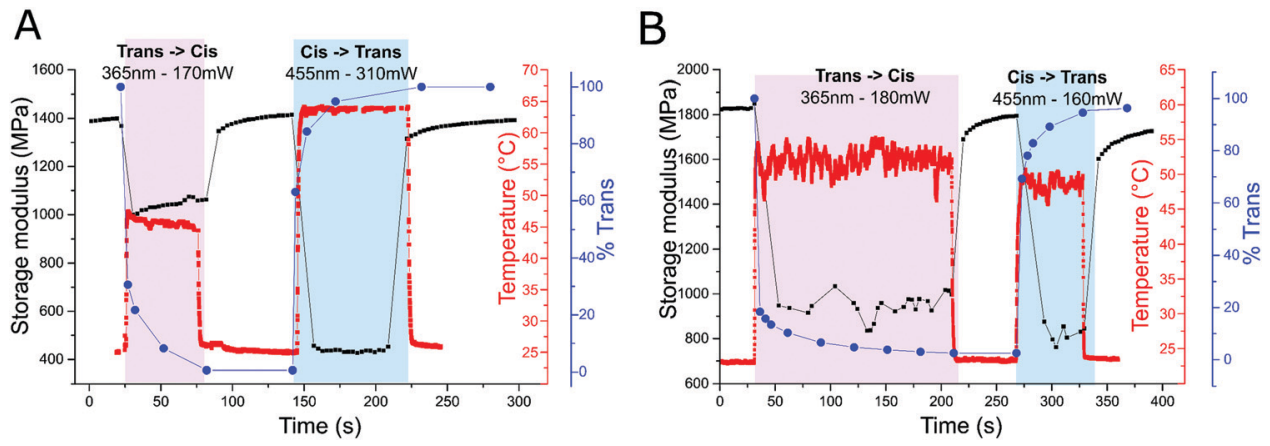

Fig. 5 (A) Overlay of variations in azobenzene isomer population, film temperature and storage modulus during illumination of splay aligned LCNs with either side-chain MY (A) or crosslinker azobenzene DY (B). The films are first illuminated with $365 \mathrm{~nm}$ light $\left(170-180 \mathrm{~mW} \mathrm{~cm}^{-2}\right)$ promoting trans-cis isomerization; upon removal of the light stimulus, both films return to their initial storage modulus. Subsequent illumination with $455 \mathrm{~nm}\left(310 \mathrm{~mW} \mathrm{~cm}^{-2}\right.$ for side-chain MY azobenzene and $160 \mathrm{~mW} \mathrm{~cm}{ }^{-2}$ for crosslinker DY) promotes cis-trans isomerization, again demonstrating a reduction of storage modulus in both polymer films. 

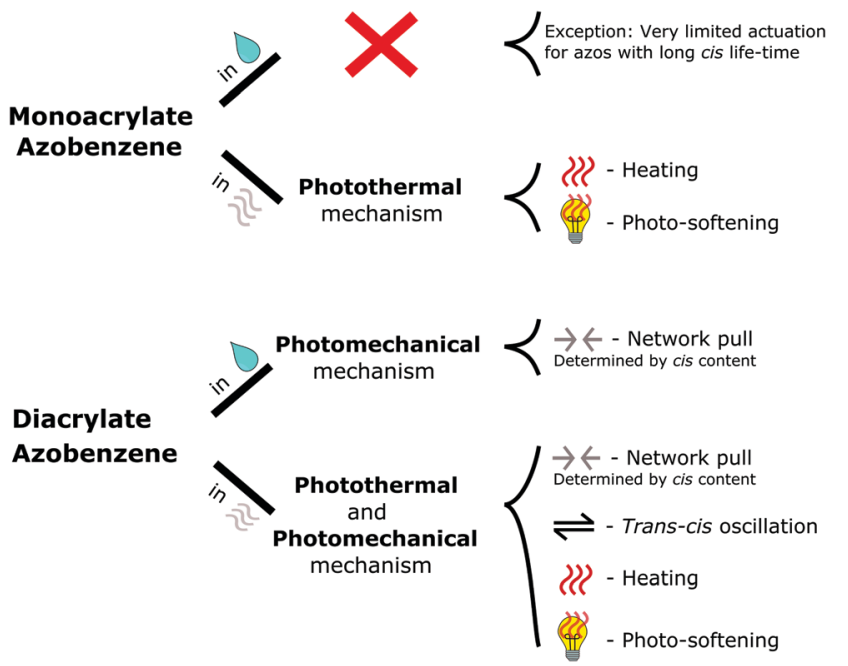

Fig. 6 Schematic roadmap for the fabrication of azobenzene doped liquid crystal actuators and to assist the distinction between the dominant factors influencing the actuation mechanism. In aqueous environment we assume an isothermal situation. The contributions involve photothermal or/and photomechanical factors depending on the nature of the azobenzene moiety and the environment of actuation.

appears to be dependent on the nature of the photo-switch, considering its connection to the network (crosslinker or as a side-chain) and the life-time of the cis isomer. In Fig. 6 we present a roadmap to assist the distinction of the dominant factors in the actuation of mono- and diacrylate chromophores in both dry and aqueous environments. Azobenzene chromophores attached to the network as side-chains with short lived cis isomers have been classified as fully photothermal-driven photo-switches, with the heat released by cis-trans isomerization fueling actuation. ${ }^{18,43}$ The photothermal nature of splay azo-MR films is accentuated by the lack of actuation underwater upon exposure to light, as submersion in water suppresses film heating.

The lack of large in-water actuation of the other monoacrylate azobenzene moiety (MY), suggests its actuation mechanism is also dominantly thermally driven. The actuation of films doped with MY-azo show negligible wavelength dependency in its actuation, further supporting a photothermal mechanism showing that the manner in which light is converted to heat (either through absorption of 365 or $455 \mathrm{~nm}$ light) has little impact. The reduced actuation in air for MY when compared to MR is due to its reduced light absorption: less energy is absorbed in the system resulting in lower film temperatures for comparable incident energies (Fig. 3B, vide supra). The negligible underwater deformation of films containing side-chain chromophores (Fig. S5, $\mathrm{ESI} \dagger$ ) suggests that the molecular disorder brought by the cis isomer configuration is not sufficient to generate large macroscopic actuation.

The remarkable underwater actuation of films doped with DY azobenzene moieties (unbending with trans-cis promoting $365 \mathrm{~nm}$ light exposure, and subsequent reversal with cis-trans promoting $455 \mathrm{~nm}$ light exposure, seen in Fig. 4C), suggests that crosslinking the chromophore to the network results in a more complex actuation mechanism. We propose aqueous actuation to be fully controlled by photomechanical deformation derived from the cis isomer populations, which generate an exerted network stress (pull-effect): this also explains the negligible light intensity dependence of deformation of DY films underwater. Isomerization kinetics show that low $365 \mathrm{~nm}$ intensity irradiation $\left(20 \mathrm{~mW} \mathrm{~cm}^{-2}\right.$ ) already converts $80 \%$ of trans states into the cis configuration, with increasing light intensities showing only slightly higher trans-cis conversion, Fig. S8 (ESI $\dagger$ ). By converting the abundant cis population into the trans isomer via exposure to $455 \mathrm{~nm}$, the films return to their initial curled state.

The same DY-azo film exposed to light in air presents a significantly different picture, with greater actuation amplitudes upon exposure to higher light intensities. The possible explanation for this and for the greater actuation efficiency of diacrylate moieties (Fig. 3A), is that the in-air actuation mechanism for DY films is likely a constructive concert between photomechanical and photothermal contributions. Our observations of the higher in-air actuation efficiency for diacrylate azobenzene moieties is in accordance with previous studies on planar aligned films that observe higher photo-generated stresses from crosslinker azobenzene compared to side-chain moieties. ${ }^{34,44}$ In contrast to the MY chromophore with long cis lifetime, films doped with DY-azo show wavelength dependent behavior when actuated in-air, with $365 \mathrm{~nm}$ illumination showing higher actuation efficiency than $455 \mathrm{~nm}$. We propose that this wavelength dependent actuation derives from photomechanical network stress (pull-effect) contributions in diacrylate chromophores. The contribution of network stress (pull-effect) is illustrated by the difference in the amplitude of actuation comparing $455 \mathrm{~nm}$ and $365 \mathrm{~nm}$ illumination in air and by the underwater actuation through $365 \mathrm{~nm}$ illumination.

However, network pull-effects are not the only contribution leading to higher deformation amplitudes in diacrylate chromophores; $455 \mathrm{~nm}$ illumination of DY films show larger deformations than the MY films with identical absorbance profile. This observation suggests that additional photomechanical contributions are present in crosslinked azobenzene moieties, and such contributions are manifested during both illumination modes (455 or $365 \mathrm{~nm}$ ). We suggest that this additional contribution is the consequence of effective molecular oscillations of the crosslinked chromophore between the two isomer configurations during illumination. ${ }^{31,45}$ This oscillation can create dynamic free volume by continuous trans-cis and cis-trans excitation, as both illumination wavelengths are absorbed by both isomeric forms. We speculate that a macroscopic manifestation of these molecular oscillations is only observable in combination with a thermal component, as no macroscopic manifestation of this effect is seen when the films are submerged in water. The importance of temperature in this photomechanical event is further illustrated by the increasing difference in the deformation amplitudes of the DY and MY azos at increasing light intensities (Fig. 3A), suggesting the contribution of this oscillation effect is more pronounced when the films heat up. We conclude that the actuation derived from diacrylate azobenzene moieties is rooted in both photothermal and photomechanical effects. Wavelength dependence is initiated by the mechanistic contribution of 


\section{Locomotion in air}
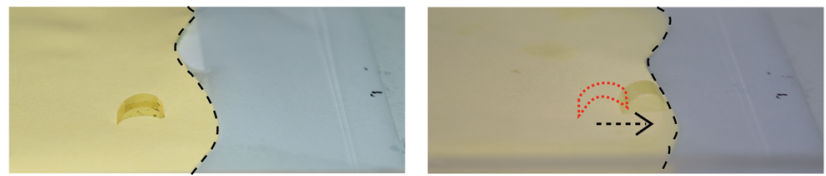

Locomotion under water
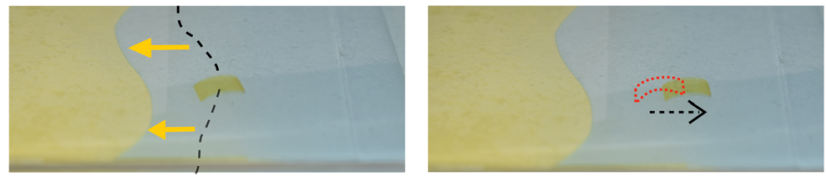

Fig. 7 Amphibious walker consisting of a single LCN film doped with 2 mol\% of the DY azobenzene. By illumination (from top), the actuator exhibits inchworm-like locomotion both in dry and aqueous environments. The locomotion in air is possible through exclusive $455 \mathrm{~nm}$ light or a combination of 365 and $455 \mathrm{~nm}$. Actuation under water is only possible through combined illumination.

network pull-effects, directly controlled by isomer population, whereas photomechanical contributions from constant molecular oscillation between cis and trans states is independent of isomer population and is directly connected to the system's temperature.

\section{Amphibious walker}

Elucidation of mechanistic contributions towards light actuation in azobenzene polymer films reveals guidelines for selecting the proper azobenzene chromophore for different applications. Monoacrylate azobenzene moieties show actuation dominated by photothermal mechanisms independent of isomer kinetics, making them excellent choices for dry environment applications with fast and reversible actuation in non-homogeneous aligned networks (such as splay) due to their relatively wavelength-independent behavior, fast reversibility and high actuation amplitude controlled through the regulation of the incident light intensity. On the other hand, applications targeted for aqueous or dual aqueous and nonaqueous application (using relatively low light intensities) will require the use of diacrylate chromophores, since actuation in water is dominated by photomechanical effects which are minimal in the monomeric azobenzenes. To illustrate the implications of our study we have created light-triggered locomotion of an inchworm-like soft robot (inspired by the design of Yamada et $a .^{20}$ ) in both dry and wet environments. The actuator moves across a paper surface when illuminated from the top, Fig. 7. When the actuator contains DY azobenzene moiety, the soft robot is not only able to walk in a dry environment (Movie S1, ESI $\dagger$ ), but when flooded with water (Movie S2, ESI $\dagger$ ), the amphibious soft robot is still able to move while submerged (albeit at a slower speed than in air), Movie S3 (ESI $\dagger$ ). Substitution of DY for monoacrylate azobenzene chromophore (MY), results in no locomotion underwater as the film shows no substantial underwater actuation.

\section{Conclusion}

In this work, we have explored the actuation efficiency of splay aligned LCN films doped with three commonly used azobenzene chromophores in air and water. Through a combined mechanical and kinetic analysis, we have uncovered the governing factors in the actuation mechanism for chromophores depending on the photo-switch chemistry, setting guidelines for future applications. We demonstrated the higher actuation efficiency of crosslinker azobenzene moieties when compared to side-chain chromophores, due to a constructive contribution between photochemical and photothermal effects in the actuation mechanism. Regardless of cis isomer life-time, side-chain chromophores predominantly actuated due to photothermal contributions, highlighting that for amplified actuation, network anisotropy provided by splay or twisted nematic alignments is of great importance. Near-zero wavelength dependence for the actuation of side-chain azobenzene photo-switches with longer cis lifetimes has been demonstrated. For crosslinked azobenzene derivatives with relatively stable cis isomers, we observed that different illumination wavelengths "activate" certain actuation pathways in which either photochemical or photothermal effects dominate. This comprehensive study was used to fabricate a primitive amphibious soft robot walker and will provide essential design rules for the choice of azobenzene chromophores for applications in the field of light responsive actuators in air and water.

\section{Conflicts of interest}

There are no conflicts to declare.

\section{Acknowledgements}

The research was made possible by a grant of Technology Foundation STW and the Impuls 2 program of the TU/e.

\section{References}

1 J. Hu, H. Meng, G. Li and S. I. Ibekwe, Smart Mater. Struct., 2012, 21, 1.

2 A. S. Hoffman, Adv. Drug Delivery Rev., 2013, 65, 10-16.

3 J. A. Lv, Y. Liu, J. Wei, E. Chen, L. Qin and Y. Yu, Nature, 2016, 537, 179-184.

4 S. I. Rich, R. J. Wood and C. Majidi, Nat. Electron., 2018, 1, 102-112.

5 H. Zeng, P. Wasylczyk, D. S. Wiersma and A. Priimagi, Adv. Mater., 2018, 30, 1-9.

6 L. Hines, K. Petersen, G. Z. Lum and M. Sitti, Adv. Mater., 2017, 29, 1-43.

7 H. K. Bisoyi, A. M. Urbas and Q. Li, Adv. Opt. Mater., 2018, 6, 1800458.

8 S. Nocentini, C. Parmeggiani, D. Martella and D. S. Wiersma, Adv. Opt. Mater., 2018, 6, 1-17.

9 J. Thévenot, H. Oliveira, O. Sandre and S. Lecommandoux, Chem. Soc. Rev., 2013, 42, 7099.

10 R. C. P. Verpaalen, M. G. Debije, C. W. M. Bastiaansen, H. Halilović, T. A. P. Engels and A. P. H. J. Schenning, J. Mater. Chem. A, 2018, 6, 17724-17729.

11 L. T. De Haan, J. M. N. Verjans, D. J. Broer, C. W. M. Bastiaansen and A. P. H. J. Schenning, J. Am. Chem. Soc., 2014, 136, 10585-10588. 
12 G. Kocak, C. Tuncer and V. Bütün, Polym. Chem., 2017, 8, 144-176.

13 A. Priimagi, A. Shimamura, M. Kondo, T. Hiraoka, S. Kubo, J. I. Mamiya, M. Kinoshita, T. Ikeda and A. Shishido, ACS Macro Lett., 2012, 1, 96-99.

14 G. N. Mol, K. D. Harris, C. W. M. Bastiaansen and D. J. Broer, Adv. Funct. Mater., 2005, 15, 1155-1159.

15 S. J. Aßhoff, F. Lancia, S. Iamsaard, B. Matt, T. Kudernac, S. P. Fletcher and N. Katsonis, Angew. Chem., Int. Ed., 2017, 56, 3261-3265.

16 S. Serak, N. Tabiryan, R. Vergara, T. J. White, R. A. Vaia and T. J. Bunning, Soft Matter, 2010, 6, 779-783.

17 N. Tabiryan, H. Koerner, K. M. Lee, T. J. White, T. J. Bunning, R. A. Vaia and M. L. Smith, Adv. Funct. Mater., 2011, 21, 2913-2918.

18 A. H. Gelebart, G. Vantomme, E. W. Meijer and D. J. Broer, Adv. Mater., 2017, 29, 1-6.

19 F. Ge, R. Yang, X. Tong, F. Camerel and Y. Zhao, Angew. Chem., Int. Ed., 2018, 57, 11758-11763.

20 M. Yamada, M. Kondo, R. Miyasato, Y. Naka, J. Mamiya, M. Kinoshita, A. Shishido, Y. Yu, C. J. Barrett and T. Ikeda, J. Mater. Chem., 2009, 19, 60-62.

21 J. J. Wie, M. R. Shankar and T. J. White, Nat. Commun., 2016, 7, 1-8.

22 X. Lu, S. Guo, X. Tong, H. Xia and Y. Zhao, Adv. Mater., 2017, 29, 1-7.

23 M. Yamada, M. Kondo, J. I. Mamiya, Y. Yu, M. Kinoshita, C. J. Barrett and T. Ikeda, Angew. Chem., Int. Ed., 2008, 47, 4986-4988.

24 H. Zeng, O. M. Wani, P. Wasylczyk and A. Priimagi, Macromol. Rapid Commun., 2017, 39, 1-6.

25 M. Rogóż, H. Zeng, C. Xuan, D. S. Wiersma and P. Wasylczyk, Adv. Opt. Mater., 2016, 4, 1689-1694.

26 S. Palagi, A. G. Mark, S. Y. Reigh, K. Melde, T. Qiu, H. Zeng, C. Parmeggiani, D. Martella, A. Sanchez-Castillo, N. Kapernaum, F. Giesselmann, D. S. Wiersma, E. Lauga and P. Fischer, Nat. Mater., 2016, 15, 647-653.

27 S. Ma, X. Li, S. Huang, J. Hu and H. Yu, Angew. Chem., Int. Ed., 2019, 2655-2659.
28 T. J. White, J. Polym. Sci., Part B: Polym. Phys., 2018, 56, 695-705.

29 K. D. Harris, R. Cuypers, P. Scheibe, C. L. van Oosten, C. W. M. Bastiaansen, J. Lub and D. J. Broer, J. Mater. Chem., 2005, 15, 5043.

30 D. Liu, C. W. M. Bastiaansen, J. M. J. Den Toonder and D. J. Broer, Angew. Chem., Int. Ed., 2012, 51, 892-896.

31 D. Liu and D. J. Broer, Nat. Commun., 2015, 6, 1-7.

32 O. M. Tanchak and C. J. Barrett, Macromolecules, 2005, 38, 10566-10570.

33 G. S. Kumar and D. C. Neckers, Chem. Rev., 1989, 89, 1915-1925.

34 A. Sánchez-Ferrer and H. Finkelmann, Macromol. Rapid Commun., 2011, 32, 671-678.

35 J. Vapaavuori, A. Laventure, C. G. Bazuin, O. Lebel and C. Pellerin, J. Am. Chem. Soc., 2015, 137, 13510-13517.

36 K. Kumar, A. P. H. J. Schenning, D. J. Broer and D. Liu, Soft Matter, 2016, 12, 3196-3201.

37 J. M. Harrison, D. Goldbaum, T. C. Corkery, C. J. Barrett and R. R. Chromik, J. Mater. Chem. C, 2015, 3, 995-1003.

38 G. J. Fang, J. E. MacLennan, Y. Yi, M. A. Glaser, M. Farrow, E. Korblova, D. M. Walba, T. E. Furtak and N. A. Clark, Nat. Commun., 2013, 4, 1-8.

39 A. H. Gelebart, D. Jan Mulder, M. Varga, A. Konya, G. Vantomme, E. W. Meijer, R. L. B. Selinger and D. J. Broer, Nature, 2017, 546, 632-636.

40 M. Poutanen, O. Ikkala and A. Priimagi, Macromolecules, 2016, 49, 4095-4101.

41 C. L. Van Oosten, K. D. Harris, C. W. M. Bastiaansen and D. J. Broer, Eur. Phys. J. E: Soft Matter Biol. Phys., 2007, 23, 329-336.

42 G. Vantomme, A. H. Gelebart, D. J. Broer and E. W. Meijer, J. Polym. Sci., Part A: Polym. Chem., 2018, 56, 1331-1336.

43 M. Pilz da Cunha, Y. Foelen, R. J. H. Van Raak, J. N. Murphy, A. P. Tom, M. G. Debije and A. P. H. J. Schenning, Adv. Opt. Mater., 2019, 1801643, 1-8.

44 M. Kondo, M. Sugimoto, M. Yamada, Y. Naka, J. I. Mamiya, M. Kinoshita, A. Shishido, Y. Yu and T. Ikeda, J. Mater. Chem., 2010, 20, 117-122.

45 K. M. Lee and T. J. White, Macromolecules, 2012, 45, 7163-7170. 